\title{
TROPHIC TRANSMISSION OF PARASITES AND HOST BEHAVIOR MODIFICATION IN DINOSAUR PREDATOR-PREY DYNAMICS
}

KURIS, Armand M., Dept. of Ecology, Evolution \& Marine Biology, University of California, Santa Barbara, CA 93106 U.S.A.

Considering the large terrestrial dinosaurs of the Jurassic and Cretaceous periods, an ecological problem is evident. Did the very large herbivores fall prey to the large carnivores? If they did, how did the large carnivores capture such large prey? A key ecological principle in extant communities is pertinent here. Across all ecosystems, even the most efficient individual predators can kill prey that weigh only about twice their own weight. The large adult sauropods apparently reached weights of up to $90,000 \mathrm{~kg}$. The largest predator, Tyrannosaurus rex weighed perhaps $6000 \mathrm{~kg}$. Could the large herbivores have been subdued by such a puny predator? Two hypotheses have been offered to address this issue. A third testable hypothesis is proposed here.

1) Social hunting extends by about one order of magnitude the size of prey that can be captured. 2) Adults of the largest sauropods may have had a refuge in size from predation. Death would have released these dinosaurs to carrion feeders. 3) I propose that large herbivorous dinosaurs were often infected with larval parasites that passed to their final hosts (carnivorous dinosaurs) via trophic transmission and that the behavior of these parasitized large herbivores was modified so as to significantly increase their susceptibility to predators. This third hypothesis is based on three ecological principles that operate in present-day predator-prey dynamics. Firstly, herbivores are often infected with larval parasites. Secondly, these parasites are usually trophically transmitted to predatory final hosts. Thirdly, these parasites often induce behavior modifications of the intermediate hosts so as to greatly increase their susceptibility to predators. All three hypotheses are potentially testable using the fossil record. Additional relevant features of parasite dynamics in dinosaur communities will be discussed. 